\section{International Scientific Journal Theoretical \& Applied Science}

Yurij Konstantinovich Dimitriev Senior Researcher Advanced Doctor in Engineering Sci., Rzhanov Institute of Semiconductor Phisics, Siberian Branch RAS, Russia dimi@isp.nsc.ru

Year: 2017 Issue: 09 Volume: 53

Published: 20.09.2017 http://T-Science.org

SECTION 4. Computer science, computer engineering and automation.

\title{
ON THE USE OF BOOLEAN FUNCTIONS FOR COMPARATIVE ANALYSIS OF MODELS OF UNRELIABLE TESTS, AND THE DEVELOPMENT OF ALGORITHMS FOR SELF-DIAGNOSIS OF MULTIPROCESSOR COMPUTING SYSTEMS
}

\begin{abstract}
Self-diagnosis of multiprocessor computing systems at system level is examined, when multiple faults occurs and under using of the unreliable tests. The state of the system is determined by the results of the comparative analysis of the outcomes of mutual testing of the system's modules. The generalization of the wellknown PMC-model of the system is used, that allows to study diagnostic properties of the system as function of properties of the used unreliable tests. For a formal description of the tests, the Boolean functions (called as consistency functions) are used, the submission of which is adapted to the solution of the self-diagnosis problem.

The set of basic (elementary) consistency functions is selected, the combination of which describes different test models from the exhaustive group of unreliable test models. The indicators of the worth of elementary consistency functions and their compositions are introduced and studied. The possibility of using of consistency functions and their worthy characteristics for comparative analysis of test outcomes, in particular, in determining the states of the modules of the system according to the fragments of the syndrome that refers to a limited neighborhood of the considered modules (the local self-diagnosis) is shown.

Key words: Local self-diagnosis on system level, theoretical-graph diagnostic model, models of unreliable test, Boolean consistency functions, worthy of consistency functions.

Language: Russian

Citation: Dimitriev YK (2017) ON THE USE OF BOOLEAN FUNCTIONS FOR COMPARATIVE ANALYSIS OF MODELS OF UNRELIABLE TESTS, AND THE DEVELOPMENT OF ALGORITHMS FOR SELF-DIAGNOSIS OF MULTIPROCESSOR COMPUTING SYSTEMS. ISJ Theoretical \& Applied Science, 09 (53): 101-112.

Soi: http://s-o-i.org/1.1/TAS-09-53-16 Doi: crossef https://dx.doi.org/10.15863/TAS.2017.09.53.16

\section{УДК 681.3.012+681.3-192 \\ ОБ ИСПОЛЬЗОВАНИИ БУЛЕВЫХ ФУНКЦИЙ ДЛЯ СРАВНИТЕЛЬНОГО АНАЛИЗА МОДЕЛЕЙ НЕНАДЕЖНЫХ ТЕСТОВ И РАЗРАБОТКИ АЛГОРИТМОВ САМОДИАГНОСТИРОВАНИЯ МНОГОПРОЦЕССОРНЫХ ВЫЧИСЛИТЕЛЬНЫХ СИСТЕМ}

Аннотация: Изучается самодиагностирование многопрочессорных вычислительных систем на системном уровне при наличии кратных неисправностей и использовании ненадежных тестов. Состояние системы определяется по результатам сопоставительного анализа исходов взаимного тестирования ее модулей. Использовано обобщение известной ПМЧ-модели, позволяющее изучать зависимость диагностических свойств системы от свойств применяемых ненадежных тестов. Для формального описания последних применяются булевы функции (функции совместности), представление которых адаптировано к решению задачи самодиагностирования. Выделено множество базовых (элементарных) функций совместности, сочетания которых описывают разные модели тестов из полной группь моделей ненадежных тестов. Введены и изучены характеристики значимости элементарных функций совместности и композиций из них для сопоставительного анализа исходов тестирования, в частности,
\end{abstract}


при определении состояния модулей системы по фрагментам синдрома, относящиися к их ограниченной окрестности (локальная самодиагностика).

Ключевые слова: локальная самодиагностика на системном уровне, графовая диагностическая модель, модели ненадежного теста, булевы функиии совместности, значимость функиий совместности.

\section{Работа выполнена при поддержке Российского фонда фундаментальных исследований (проект № 14-07-00169.a).}

\section{Introduction}

Рассматривается возможность автоматизированной диагностики (самодиагностики) масштабируемых живучих многопроцессорных вычислительных систем, основанной на сопоставительном анализе исходов взаимного тестирования ее процессорных модулей (далее просто модулей), осуществляемого самой диагностируемой системой с помощью приданных модулям системы программноаппаратурных средств. Для изучения проблем самодиагностики используется теоретикографовая модель, предложенная в работе [1] и известная как ПМЧ-модель. Эта модель описывает диагностику вычислительной системы при кратных неисправностях, рассматриваемых на уровне модулей (системная диагностика), при использовании ненадежных тестов. Последнее означает, что оценка, получаемая исправным тестирующим модулем, соответствуют фактическому состоянию тестируемого модуля, а оценка, получаемая неисправным тестирующим модулем, может не соответствовать ему.

Для управления диагностированием живучих многопроцессорных вычислительных систем используют децентрализованные адаптивные алгоритмы. В [2, 3] для ПМЧ-модели предложен подход к самодиагностике, который базируется на определении состояния каждого модуля системы по результатам выполняемого им сопоставительного анализа подмножества оценок, получаемых в реальном времени и относящихся к ограниченной окрестности этого модуля (локальная самодиагностика).

ПМЧ-модель представляет собой лишь одну из возможных моделей ненадежных тестов, а именно, модель, в которой оценка, получаемая неисправным тестирующим модулем, не зависит от фактического состояния тестируемого модуля. В работе [4] перечислены другие возможные модели полных ненадежных тестов. В работе [5] выделено базовое множество булевых функций (элементарных функций совместности), с помощью которых можно описать все модели ненадежных тестов, составляющих исчерпывающую группу [4]; установлена взаимосвязь между моделями этой группы.

Цель работы - изучить диагностические свойства введенных в [5] элементарных функций совместности и возможность их применения для сравнительного анализа моделей ненадежных тестов и для локальной идентификации состояния модулей в рамках этих моделей.

Статья состоит из введения и пяти разделов. В разделе 2 дана постановка решаемой в статье задачи. Раздел 3 дает общее представление о процессе локального самодиагностирования, которое основано на подходе, предложенном в [2]. В разделе 4 даны основные определения и введены критерии значимости булевых функций совместности для решения задач самодиагностики. Значимость функции совместности выражается в возможности выявить присутствие и взаимное расположение неисправных и / или исправных модулей в описываемой ею (ограниченной) области системы. В разделе 5 изучены свойства значимости функций совместности для разных моделей ненадежных тестов. В разделе 6 подведены итоги выполненного исследования.

В связи с широкой распространенностью ПМЧ-модели и с целью сокращения объема статьи описание основных понятий самодиагностики при кратных неисправностях и ненадежных тестах опущено. Использованы терминология и нотация, введенные в [2, 3]; краткий список используемых обозначений приведен в Приложении. Для упрощения изложения терминология системы распространена на представляющие ее модели.

\section{Problem statement}

Согласно [5], обобщенная модель самодиагностируемой системы представлена двойкой $\langle D, A\rangle$, где диагностический граф $D-$ модель структуры (тестовых) связей между модулями системы, по которым одни модули могут проверить состояние других модулей, и $\langle A\rangle$ - модель теста, используемого для самодиагностирования. Четверка

$$
<A>=<a(g, g) a(g, b) a(b, g) a(b, b)>
$$

булевых переменных перечисляет значения исхода тестирования при всех возможных состояниях тестирующего и тестируемого модулей (соответственно первый и второй символы в круглой скобке); $g$ - исправный модуль, $b$ - неисправный модуль. Каждая переменная принимает одно из трех значений: 0 , 1 или х, где 0 или 1 означает, что тестирующий модуль оценивает состояние тестируемого как исправное или неисправное соответственно, а символ "х" соответствует непредсказуемому (0 
или 1) результату оценки. Ниже для моделей тестирования используем обозначения $M_{a(g, g) a(g, b) a(b, g) a(b, b)}$.

Рассматривается

самодиагностика, основанная на использовании моделей тестов, описанных в [4], для случая устойчивых отказов модулей системы. Группу [4] составляют модели, представляющие полные тесты. Если тест выполняется исправным тестирующим модулем, то его оценка состояния тестируемого модуля достоверна (что вытекает из свойства полноты теста). Если тестирующий модуль неисправен, то оценка, которую он дает состоянию тестируемого модуля, может соответствовать или не соответствовать фактическому состоянию последнего. Это происходит в результате утраты свойства полноты теста или неверной интерпретации результатов тестирования. Тесты, обладающие описанными свойствами, называют полными, но ненадежными, или просто ненадежными [1].

Модели полных ненадежных тестов из группы [4] имеют описание вида $M_{01 a(b, g) a(b, b)}$, а группа моделей в целом описывается в виде: $M_{01 z z}$, где $z \in\{0,1, \mathrm{x}\}$. Группа $M_{01 z z}$ состоит из девяти моделей, представляющих все возможные сочетания значений пары переменных $\{z, z\}^{1}$.

В статье рассматривается одна из трех основных задач самодиагностики - задача самодиагностирования. Она формулируется следующим образом. Заданы диагностический граф $D=(V, E)$, модель ненадежных тестов $<A>$ и множество допустимых образов неисправностей $F(t)$. Для заданного синдрома $\sigma$, основываясь на зависимости исхода тестов от состояния участвующих в нем вершин, описываемой четверкой $\langle A\rangle$, определить такой образ неисправностей $F$, порождающий синдром $\sigma$, что $F \in F(t)$.

Решение задачи самодиагностирования состоит в разработке алгоритма, с помощью которого осуществляется управление последовательностью шагов тестирования, сбора и сопоставительного анализа полученных исходов тестирования. Эффективность алгоритма самодиагностирования оценивают числом $T$ тестов, выполнение которых необходимо для

1 Особое место в группе занимает модель $M_{0101}$ так называемого "совершенного тестера", когда тестирующая вершина верно оценивает состояние тестируемой независимо от своего состояния. Эта модель включена в группу моделей ненадежных тестов только из соображений полноты. определения технического состояния всех модулей системы. Значение $T$ находится в прямой зависимости от эффективности используемых правил сопоставительного анализа. Эти правила задают взаимно однозначное соответствие между значением синдрома и фактическим состоянием некоторых модулей системы, участвующих в его формировании.

Работа посвящена поиску правил сопоставительного анализа исходов тестирования при локальном самодиагностировании масштабируемых живучих многопроцессорных вычислительных систем, осуществляемом с помощью децентрализованных адаптивных алгоритмов [2, 3, 6, 7]. В качестве формального аппарата для решения задачи сопоставительного анализа используются булевы функции, называемые функциями совместности. В [5] выделено базовое множество (элементарных) функций совместности, с помощью которых описывается исчерпывающее множество (группа) моделей ненадежных тестов [4]. В [5] введены и исследованы критерии значимости функций совместности в отношении возможности идентифицировать или предсказывать техническое состояние модулей, соответствующих ее переменным.

В данной работе анализ свойств значимости элементарных функций совместности осуществляется с позиций теории множеств. Изучаются 1) отношения порядка модулей, участвующих в тестировании, по их техническому состоянию и 2 ) возможность использования выявленных отношений для анализа значимости функций совместности, представляющих собой композиции заданных элементарных функций совместности.

\section{Markup of the diagnostic graph}

В $[2,3,6,6]$ решение задачи самодиагностирования моделируется как разметка диагностического графа. Для выполнения разметки каждой вершине $v$ диагностического графа присвоена метка $m(v) \in M ;$ в простейшем случае множество $M=\{0,1,2\}$. Значение $m(v)=2$, если фактическое состояние вершины $v$ не идентифицировано (в частности, перед началом диагностирования); значение $m(v)=0$ или $m(v)=1$, если по результатам анализа текущего фрагмента синдрома $\sigma$ вершина $v$ признана соответственно исправной или неисправной (эти состояния называем финальными). Разметка 
графа завершается, когда установлено финальное состояние для всех его вершин ${ }^{2}$.

При выполнении разметки поведение каждой вершины графа зависит от состояния смежных с нею вершин, что соответствует принципу близкодействия, характерному для клеточных автоматов [8, 9].

Для управления клеточным автоматом используют децентрализованные алгоритмы, в основу которых положены три ключевых требования: 1) отсутствие сведений о глобальном состоянии системы, 2) отсутствие глобальной шкалы времени и 3) недетерминизм. Эти требования при использовании развиваемого в [2, $3,6,7]$ подхода реализуются тем, что 1) фактическое состояние каждого модуля системы определяется путем сопоставительного анализа исходов тестирования, которые относятся только к модулям из его ограниченной (но допускающей рост в ходе диагностирования) окрестности; 2) сопоставительный анализ фрагмента синдрома, полученного на текущем шаге самодиагностирования, производится с учетом достигнутой к этому шагу разметки графа; 3) для принятия модулем решения важен не порядок формирования фрагмента синдрома, а его состав по числу и значению соответствующих исходов тестирования.

Используемые для сопоставительного анализа соотношения между исходами тестирования и состоянием соответствующих вершин называем правилами (локального) самоопределения. Последствия локального самоопределения вершин состоят в следующем. 1. Идентификация исправной вершины $v$ позволяет определять финальное состояние любой вершины $w \in \Gamma(v)$ по исходу единственного теста $(v, w)$.

2. Идентификация неисправной вершины дает возможность в ходе диагностирования корректировать аксиому о кратности допустимых неисправностей. Аксиома о кратности ограничивает сверху число одновременно неисправных вершин графа (следовательно, и общее число допустимых состояний графа) заданным значением $t, t \leq N-1$, называемым кратностью неисправностей. Идентификация $\square$ неисправных вершин соответственно уменьшает значение $t$. Уменьшение кратности

2 Термин разметка графа применяем как к процессу определения финального состояния вершин графа, так и к описанию множества вершин графа, получивших метки финального состояния на некотором шаге самодиагностирования. неисправностей ведет к сокращению времени разметки диагностического графа.

Идентифицированные неисправные вершины исключаются из диагностического графа вместе с инцидентными им дугами. Обозначим $F^{(r)}-$ множество неисправных вершин, идентифицированных на $r$-м шаге разметки. В таком случае правила самоопределения для вершины $v$ применяются к (остаточному) множеству вершин графа, включающему в себя подмножество $H^{(r)}(v)=H^{(r-1)}(v)-\left[F^{(r)} \cap H^{(r-1)}(v)\right] \quad$ вершин, смежных с вершиной $v$ в диагностическом графе $D$, финальное состояние которых еще не определено. Правила самоопределения применяются, исходя из достигнутого значения $Q^{(r)}(v)=Q^{(r-1)}(v)-\left|F^{(r)} \cap H^{(r-1)}(v)\right|-$ локально оцениваемого для вершины $v$ наибольшего возможного числа еще не идентифицированных неисправных вершин в остаточном диагностическом графе ${ }^{3}$. Величину $Q^{(r)}(v)$ называем порогом самоопределения, а значение $\left|F^{(r)} \cap H^{(r-1)}(v)\right|=\tau^{(r)}(v) \quad-\quad$ величиной корректировки порога самоопределения для вершины $v$. Начальное значение порога самоопределения для всех вершин графа равно принятой кратности неисправностей: $\forall v \in V\left\{Q^{(0)}(v)=t\right\}$. В ходе диагностирования порог самоопределения для каждой вершины соответствует числу учтенных ею идентифицированных неисправных вершин.

Пример формального описания и доказательства полноты и корректности децентрализованного адаптивного алгоритма локального самодиагностирования дан в [3] для случая ПМЧ-модели ненадежных тестов. Алгоритм базируется на реализации описанной в этом разделе разметки графа и использует множество введенных автором условий самоопределения.

\section{Unreliable test models and consistency functions}

\subsection{Elementary functions of consistency}

Пусть структурированная модель самодиагностируемой системы представлена двойкой $<D, A>$. Модель $<A>$ ненадежного теста называем детерминированной, если ни один из элементов представляющей ее четверки $\langle A>$

3 При $r=0$ для каждой вершины $v \in V$ диагностического графа выполняется $H^{(0)}(v)=H(v)$. 
не принимает значения “х”, и недетерминированной в противном случае.

Пусть $D=(V, E)$ - диагностический граф и $(v, w) \in E$. Применительно к произвольным вершинам $v, w \in V$, таким, что $(v, w) \in E$, модель теста удобно описывать в виде $<A>=<a_{v w} a_{v w} a_{-w} a_{--}>$. Здесь прямая форма индексной переменной соответствует исправности, а инверсная форма неисправности соответствующей вершины, участвующей в выполнении теста $(v, w)$. Если тест $(v, w)$ выполнен, то его исход $z$ принимает значение 0 , когда вершина $v$ считает, что вершина $w$ исправна, и значение 1 в противном случае. Назовем подграфом тестирования подграф $D_{T}=\left(V_{T}, E_{T}\right)$ диагностического графа, индуцированный множеством его взвешенных дуг, т.е. дуг, соответствующих тестам, которые выполнены к текущему шагу разметки. В подграфе тестирования можно выделить (возможно, вершинно пересекающиеся) zподграфы $\quad D_{T(z)}=\left(V_{T(z)}, E_{T(z)}\right), \quad z \in\{0,1\}$, индуцированные соответственно дугами веса 0 и 1. Для заданного значения $z$ подграф тестирования $D_{T(z)}$ состоит из $K_{z}$ компонент $z-$ связности, $K_{z} \geq 0$, для которых используем обозначение $D_{z}^{(i)}=\left(V_{z}^{(i)}, E_{z}^{(i)}\right), i=\overline{0, K_{z}}$. Здесь и ниже любая компонента $z$-связности рассматривается как компонента слабой связности. Будем обозначать $D_{z}=\left(V_{z}, E_{z}\right)$ и называть $z$-подграфом - произвольный связный подграф графа $D_{T(z)}=\left(V_{T(z)}, E_{T(z)}\right)$.

О п р е д е л е и е 1 . Элементарной функцией z-совместности называется функция вида

$$
f_{z}(v, w)=\bigvee\left(v^{p} w^{p} \mid a_{v^{p} w^{p}}=z\right),
$$

описывающая состояние вершин $\{v, w\}$, при которых исход $a(v, w)$ теста $(v, w)$ принимает заданное значение $z \in\{0,1\}$ для заданной модели теста $<A>$. Здесь $p \in\{0,1\}$, так что $u^{0}=u, u^{1}=\bar{u}, u \in\{v, w\}$.

Модель $<A>$ ненадежного теста описывается парой элементарных функций совместности $f_{z}(v, w), \quad z \in\{0,1\} . \quad$ В табл. 1 приведены элементарные функции $z$-совместности для группы моделей ненадежных тестов [4], полученные в [5]. Для справки в табл. 1 приведены достигаемые для заданных моделей значения кратности неисправностей.

Элементарные функции $z$-совместности (ЭФС).

Таблица 1

\begin{tabular}{|c|c|c|c|}
\hline Модель & ЭФС & Вид ЭФС & Кратность неисправностей \\
\hline 1 & 2 & 3 & 4 \\
\hline \multirow{2}{*}{$M_{01 \mathrm{xx}}$} & $f_{0}(v, w)=\bar{v} \vee w$ & $0-\mathrm{I}$ & \multirow{4}{*}{$t \leq\lfloor(N-1) / 2\rfloor$} \\
\hline & $f_{1}(v, w)=\bar{v} \vee \bar{w}$ & $1-\mathrm{I}$ & \\
\hline \multirow{2}{*}{$M_{01 \times 0}$} & $f_{0}(v, w)=\bar{v} \vee w$ & $0-\mathrm{I}$ & \\
\hline & $f_{1}(v, w)=v \bar{w} \vee \bar{v} w$ & $1-\mathrm{II}$ & \\
\hline \multirow{2}{*}{$M_{01 \times 1}$} & $f_{0}(v, w)=w$ & 0 -II & \multirow{2}{*}{$t \leq N-2$} \\
\hline & $f_{1}(v, w)=\bar{v} \vee \bar{w}$ & $1-\mathrm{I}$ & \\
\hline \multirow{2}{*}{$M_{010 \mathrm{x}}$} & $f_{0}(v, w)=\bar{v} \vee w$ & $0-\mathrm{I}$ & \multirow{2}{*}{$t \leq N-1$} \\
\hline & $f_{1}(v, w)=\bar{w}$ & 1 -III & \\
\hline \multirow{2}{*}{$M_{011 \mathrm{x}}$} & $f_{0}(v, w)=v w \vee \bar{v} \bar{w}$ & 0 -III & \multirow{2}{*}{$t \leq\lfloor(N-1) / 2\rfloor$} \\
\hline & $f_{1}(v, w)=\bar{v} \vee \bar{w}$ & $1-\mathrm{I}$ & \\
\hline \multirow{3}{*}{$M_{0100}$} & $f_{0}(v, w)=\bar{v} \vee w$ & $0-\mathrm{I}$ & \multirow{2}{*}{$t \leq N-1$} \\
\hline & $f_{1}(v, w)=v \bar{w}=\overline{f_{0}(v, w)}$ & $1-\mathrm{IV}$ & \\
\hline & $f_{0}(v, w)=v w \vee \bar{v} \bar{w}$ & 0 -III & \\
\hline
\end{tabular}




\begin{tabular}{|c|c|c|c|c|c|c|}
\hline Impact Factor: & $\begin{array}{l}\text { ISRA (India) } \\
\text { ISI (Dubai, UAF } \\
\text { GIF (Australia) } \\
\text { JIF }\end{array}$ & $\begin{array}{l}=1.344 \\
=0.829 \\
=0.564 \\
=1.500\end{array}$ & $\begin{array}{l}\text { SIS (USA) } \\
\text { PИНЦ (Russia) } \\
\text { ESJI (KZ) } \\
\text { SJIF (Morocco) }\end{array}$ & $\begin{array}{l}=0.912 \\
=0.207 \\
=\mathbf{3 . 8 6 0} \\
=\mathbf{2 . 0 3 1}\end{array}$ & $\begin{array}{l}\text { ICV (Poland) } \\
\text { PIF (India) } \\
\text { IBI (India) }\end{array}$ & $\begin{array}{l}=6.630 \\
=1.940 \\
=4.260\end{array}$ \\
\hline
\end{tabular}

\begin{tabular}{|c|c|c|c|}
\hline$M_{0110}$ & $f_{1}(v, w)=v \bar{w} \vee \bar{v} w$ & $1-$ II & $t \leq\lfloor(N-1) / 2\rfloor$ \\
\hline \multirow{2}{*}{$M_{0111}$} & $f_{0}(v, w)=v w$ & 0 -IV & $t \leq N-2$ \\
\cline { 2 - 3 } & $f_{1}(v, w)=\bar{v} \vee \bar{w}$ & $1-\mathrm{I}$ & \\
\hline \multirow{2}{*}{$M_{0101}$} & $f_{0}(v, w)=w$ & $0-\mathrm{II}$ & \multirow{2}{*}{$N$} \\
\cline { 2 - 3 } & $f_{1}(v, w)=\bar{w}$ & 1 -III & \\
\hline
\end{tabular}

Заметим, что для недетерминированных моделей тестов элементарные функции zсовместности являются неполностью определенными булевыми функциями, доопределяемыми значением 0 для состояний, которые не совместны с заданным исходом тестирования $z$.

Как видно из табл. 1, для рассматриваемой группы моделей имеется по четыре вида для элементарных функций 0- и 1-совместности.

\subsection{Z-consistency functions}

Пусть $F$ - образ неисправностей, $\sigma^{(r)}(F)$ - фрагмент порождаемого им синдрома ${ }^{4}$, который сформирован после $r$-го шага разметки, $\sigma^{(r)}(F) \subset \sigma(F)$, и $D_{T}$ - подграф тестирования, индуцируемый элементами $\sigma^{(r)}(F)$.

О п р е д е л е и е 2. Для фрагмента синдрома $\sigma^{(r)}(F)$ функцией совместности называется конъюнкция элементарных функций $z$-совместности, соответствующих значениям элементов из $\sigma^{(r)}(F)$, и приведенная к совершенной дизъюнктивной нормальной форме.

О п р е д е ле н и е 3. Редуциированной называем функцию совместности, минтермы которой содержат не более $Q^{(r)}\left(D_{T}\right)$ переменных в инверсной форме. Здесь $Q^{(r)}\left(D_{T}\right)$ - порог самоопределения, рассчитанный на $r$-ом шаге разметки для подграфа $D_{T}$.

Любую функцию, полученную в результате тождественных преобразований редуцированной функции совместности, также называем редуцированной.

О п р е д е л е и е 4. Определяющей называется редуцированная функция совместности, если она имеет хотя бы одну общую переменную в прямой или инверсной форме. Выделенные общие переменные (равно

4 Фрагментом синдрома называем упорядоченное подмножество элементов синдрома, имеющих значение 0 или 1 и соответствующих множеству тестов, выполненных к текущему такту разметки. как и вершины диагностического графа, которым они сопоставлены) называем определимыми.

Описанные в разделе 3 последствия идентификации финального состояния вершин представляют собой проявления свойств определимости редуцированной функции совместности, общие для всех моделей ненадежных тестов.

О п р е д е л е н и е 5. Продуктивной называется редуцированная функция совместности, если ее форма позволяет уточнить (или ограничить) область размещения исправных или неисправных вершин, их число или взаимное расположение.

Свойство продуктивности редуцированной функции совместности, описывающей подграф тестирования $D_{T}=\left(V_{T}, E_{T}\right)$ указывает на возможность (и способы) получения информации, способствующей идентификации финального состояния вершин из $V_{T}$ (или уменьшению общего числа тестов, необходимых для разметки диагностического графа), в результате выполнения тестов с участием вершин из $V_{T}$.

Функции совместности, удовлетворяющие определениям 4 и 5, называем значимыми. Наличие или отсутствие свойств значимости заданной функции $z$-совместности ассоциируем с неформальным понятием «диагностические свойства ${ }^{5} »$ соответствующего подграфа тестирования. Результаты анализа значимости редуцированных функций совместности могут быть использованы для разработки эффективных алгоритмов самодиагностирования.

П р и м р 1. Для модели $M_{011 x}$ элементарная функция 0-совместности $f_{0}(v, w)=v w \vee \bar{v} \bar{w} \quad$ продуктивна, когда локальный порог определимости для $\{v, w\}$ не

5 Под диагностическими свойствами понимаем любые количественные или качественные показатели, которые позволяют оценить достижимость или эффективность самодиагностирования, a также признаки, которые позволяют идентифицировать состояние отдельных модулей системы. 
менее 2, а при локальном пороге определимости равном единице становится определимой: $f_{0}(v, w)=v w, \quad$ то есть идентифицирует исправность вершин $v$ и $w$.

Поскольку идентификация финального состояния вершин диагностического графа осуществляется в результате сопоставительного анализа исходов тестирования, каждой компоненте $z$-связности подграфа тестирования соответствует отдельная функция совместности. Поэтому, если не оговорено другое и без нарушения общности, в качестве подграфа тестирования рассматриваем только связные $z$ подграфы.

Наблюдаемое в табл.1 совпадение видов элементарных функций $z$-совместности для разных моделей ненадежных тестов дает основание делить модели на группы. Если модели тестирования имеют одну и ту же элементарную функцию $z$-совместности, то для них диагностические свойства любого выделенного $z$-подграфа, совпадают. Понятие "диагностические свойства" $z$-подграфа в данном случае означает, что для каждой из моделей тестов, имеющих общую элементарную функцию $z$-совместности $f_{z}$, в любом заданном $z$-подграфе $D_{z}=\left(V_{z}, E_{z}\right)$ состояние одноименных вершин из $V_{z}$ эквивалентно. Очевидно также выполнение обсуждаемого свойства для объединения любых подграфов $D_{z}^{(i)}, D_{z}^{(j)} \in D_{T}$.

\section{Binary relations and elementary functions of $z$-consistency}

Известна связь между теорией множеств и булевой алгеброй. Она выражается в возможности заменить теоретико-множественные операции над системой подмножеств данного множества поразрядными логическими операциями над соответствующими двоичными векторами. Теоретико-множественный подход позволяет изучать взаимную зависимость состояний вершин в компонентах связности подграфа тестирования, связываемую со свойствами ${ }^{6}$ симметричности и транзитивности бинарных отношений эквивалентности и порядка.

\subsection{Symmetry of elementary functions of $z-$} consistency

Впервые понятие "симметричность" было введено для характеристики зависимости исхода ненадежного теста от состояния тестируемой вершины в недетерминированных моделях

6 Слово "свойства" используется в смысле характеристики, которой может обладать или не обладать каждый объект множества. ненадежных тестов [4]. В работах [9, 10] понятие симметричности распространено на детерминированные модели.

В данной работе используется более дифференцированный подход к определению симметричности модели ненадежных тестов, основанный на свойствах представляющих ее элементарных функций z-совместности.

Существует несколько эквивалентных определений понятия симметрии булевой функции. Конструктивным является подход [10], при котором предварительно выявляется симметрия пар аргументов $x_{i}$ и $x_{j}$ булевой функции (аргументы $x_{i}$ и $x_{j}$ симметричны, если при их перестановке значение функции не изменяется), и лишь затем выявляется симметрия функции (функция обладает полной симметрией, если все ее аргументы симметричны).

Обозначим $f\left(x_{1}, x_{2}, \ldots x_{i}, \ldots x_{j}, \ldots, x_{n}\right)$ булеву функцию от переменных $\left\{x_{1}, x_{2}, \ldots x_{i}, \ldots x_{j}, \ldots, x_{n}\right\}$. Выявление симметрии пар аргументов базируется на сопоставлении двух кофакторов булевой функции. Кофактором функции $f\left(x_{1}, x_{2}, \ldots x_{i}, \ldots x_{j}, \ldots, x_{n}\right)$ по переменным $x_{i}$ и $x_{j}$ называют остаточную подфункцию, образующуюся при подстановке в $f$ заданных значений для переменных $x_{i}$ и $x_{j}$. Для любой пары переменных $\left\{x_{i}, x_{j}\right\}$ в полностью определенной булевой функции $f\left(x_{1}, x_{2}, \ldots x_{i}, \ldots x_{j}, \ldots, x_{n}\right)$ можно выделить четыре кофактора:

$$
\begin{aligned}
& f_{x_{i} x_{j}}=f\left(x_{1}, x_{2}, \ldots 0, \ldots 0, \ldots, x_{n}\right), \\
& f_{x_{i} \overline{x_{j}}}=f\left(x_{1}, x_{2}, \ldots 0, \ldots 1, \ldots, x_{n}\right), \\
& f_{\overline{x_{i} x_{j}}}=f\left(x_{1}, x_{2}, \ldots 1, \ldots 0, \ldots, x_{n}\right), \\
& f_{\bar{x}_{i} \bar{x}_{j}}=f\left(x_{1}, x_{2}, \ldots 1, \ldots 1, \ldots, x_{n}\right) .
\end{aligned}
$$

Согласно соотношениям кофакторов, выделяют: прямую неэквивалентную симметрию $x_{i} N x_{j} \quad$ и прямую эквивалентную симметрию $x_{i} E x_{j}$, а также смешанную неэквивалентную и смешанную эквивалентную симметрию, обозначаемую соответственно $x_{i} ! N x_{j}$, и $x_{i} ! E x_{j}$. Эти четыре типа симметрии переводятся соответственно в следующие требования к кофакторам заданной функции: $f_{01}=f_{10}$ или, в эквивалентной форме, $f_{01} \oplus f_{10}=0 ; f_{00}=f_{11}$, или $f_{00} \oplus f_{11}=0 ; \quad f_{01}=\overline{f_{10}}, \quad$ или $\quad f_{10} \oplus f_{01}=1 ;$ $f_{00}=\overline{f_{11}}$, или $f_{00} \oplus f_{11}=1$. Здесь $\oplus-$ знак суммирования по модулю 2. Функция $f$ имеет 


\section{Impact Factor:}

\begin{tabular}{ll|lr} 
ISRA $($ India & $=\mathbf{1 . 3 4 4}$ & SIS $($ USA) & $=\mathbf{0 . 9 1 2}$ \\
ISI $($ Dubai, UAE) $=\mathbf{0 . 8 2 9}$ & PИНЦ (Russia) $=\mathbf{0 . 2 0 7}$ \\
GIF $($ Australia) $=\mathbf{0 . 5 6 4}$ & ESJI $($ KZ) & $=\mathbf{3 . 8 6 0}$ \\
JIF & $=\mathbf{1 . 5 0 0}$ & SJIF $($ Morocco $)=\mathbf{2 . 0 3 1}$
\end{tabular}

прямую полиморфную симметрию $x_{i} M x_{j}$, если одновременно выполняется $x_{i} N x_{j}$ и $x_{i} E x_{j}$, что влечет выполнение равенств $f_{01}=f_{10}$ и $f_{00}=f_{11}$. Аналогично определяется смешанная полиморфная симметрия $x_{i} ! M x_{j}$.

Характеристика симметричности ЭФС.

Таблица 2

\begin{tabular}{|c|c|c|c|c|c|c|c|}
\hline \multirow{2}{*}{\multicolumn{2}{|c|}{ ЭФС }} & \multicolumn{4}{|c|}{ Тип симметричности } & \multirow[t]{2}{*}{ Изотропность } & \multirow{2}{*}{$\begin{array}{c}\text { Модель } \\
\text { тестирования }\end{array}$} \\
\hline & & $v E w$ & $v N w$ & $v ! E w$ & $v ! N w$ & & \\
\hline 1 & 2 & 3 & 4 & 5 & 6 & 7 & 8 \\
\hline $0-\mathrm{I}$ & $f_{0}(v, w)=\bar{v} \vee w$ & \multirow{2}{*}{+} & & & \multirow{2}{*}{+} & $A n I_{0}$ & \multirow{2}{*}{$M_{0100}$} \\
\hline 1-IV & $f_{1}(v, w)=v \bar{w}$ & & & & & $A n I_{I}$ & \\
\hline 0 -II & $f_{0}(v, w)=w$ & & & \multirow{2}{*}{+} & \multirow{2}{*}{+} & $A n I_{0}$ & \multirow{2}{*}{$M_{0101}$} \\
\hline 1-III & $f_{1}(v, w)=\bar{w}$ & & & & & $A n I_{I}$ & \\
\hline 0-III & $f_{0}(v, w)=v w \vee \bar{v} \bar{w}$ & \multirow{2}{*}{+} & \multirow{2}{*}{+} & & & $I_{0}$ & \multirow{2}{*}{$M_{0110}$} \\
\hline 1 -II & $f_{1}(v, w)=v \bar{w} \vee \bar{v} w$ & & & & & $I_{1}$ & \\
\hline 0-IV & $f_{0}(v, w)=v w$ & & \multirow[b]{2}{*}{+} & \multirow[b]{2}{*}{+} & & $I_{0}$ & \multirow{2}{*}{$M_{0111}$} \\
\hline $1-\mathrm{I}$ & $f_{1}(v, w)=\bar{v} \vee \bar{w}$ & & & & & $I_{1}$ & \\
\hline
\end{tabular}

В табл. 2 (столбцы 3-6) приведены характеристики симметричности элементарных функций $z$-совместности. Наличие свойства симметричности в таблице отмечено знаком "+". Как и везде в этой работе, при составлении таблицы значений функции $z$-совместности, которая необходима для определения типа симметрии функции, прямое значение переменной (например, значение $v^{\prime}=0^{7}$ ) указывает, что соответствующая вершина (v) исправна.

Из табл. 2 видно, что по характеру симметрии элементарные функции zсовместности объединяются в группы. Так, по типу симметрии они объединяются в пары функций, которые дополнительны друг по отношению к другу. Заметим, что эти пары соответствуют детерминированным моделям ненадежного тестирования.

По свойству неэквивалентной симметрии элементарные функции z-совместности делятся на две группы. Функции 0-III, 0-IV, 1-I, 1-II образуют группу функций с прямой неэквивалентной симметрией $(v N w)$; функции 0-I, 0-II, 1-III,1-IV составляют группу функций со смешанной неэквивалентной симметрией $(v ! N w)$.

Элементарные функции $z$-совместности перечисляют состояния пары вершин-участников

${ }^{7}$ Символ $x^{\prime}$ указывает значение переменной $x, x^{\prime} \in\{0,1\}$. теста, связанных отношением порядка, или их роли в тесте (тестирующая-тестируемая). В соответствии с этим симметричность функции означает инвариантность рассматриваемого исхода тестирования $z$ к роли вершин в тестировании: $f_{z}(v, w)=f_{z}(w, v)$. Анализ этого отношения представляет интерес для выявления значимости взаимного тестирования между смежными вершинами. В принятой типизации свойству коммутативности отвечают элементарные функции $z$-совместности с прямой неэквивалентной симметрией и вне зависимости от характера эквивалентной симметрии.

Таким образом, свойство прямой неэквивалентной симметрии применительно к элементарной функции $z$-совместности характеризует особый вид отношения симметрии, которому в физике соответствует понятие изотропности. Изотропность означает одинаковость физических свойств во всех направлениях, то есть симметрию по выбору направления. Неэквивалентная смешанная симметрия характеризует свойство анизотропности. Применительно к диагностике термин "изотропность" представляется более предпочтительным, нежели "симметрия", поскольку характеризует отношение порядка между вершинами-участниками теста $(v, w)$.

О п р е д е л е н и е $\quad 6 . \quad$ Элементарная функция z-совместности называется изотропной, если она содержит конституенты единицы, в которых переменные имеют одинаковую 
(инверсную или неинверсную) форму и/или пару конституент единицы с переменными, имеющими разные знаки инверсии. Функцию, не обладающую свойством изотропности, называем анизотропной.

Согласно значению $z$ исхода тестирования, различаем 0- и 1-изотропность и 0 - и 1 анизотропность функций совместности. Свойство изотропности $I_{z}$ и анизотропности $A n I_{z}$ элементарных функций $z$-совместности указано в столбце 7 табл. 2 .

С в о й с т в о 1 . При использовании изотропной элементарной функции zсовместности функция совместности любого $z$ подграфа тестирования $D_{z}$ не зависит от направления его дуг.

Тождественность функций $z$-совместности для подграфов тестирования $D_{z}=\left(V_{z}, E_{z}\right)$, указываемая свойством 1 , предоставляет возможность для каждого выполненного теста $(v, w) \in E_{z} \quad$ оценить целесообразность выполнения обратного теста $(w, v)$, исходя из учета возможности разных значений его исхода: $a(w, v)=z \quad$ или $\quad a(w, v)=\bar{z} . \quad$ Подобную "прогностическую" возможность можно рассматривать как выражение продуктивности отношения изотропности.

Свойство изотропности находится в аккордансе с применением для построения многопроцессорных вычислительных систем дуплексных межмодульных связей, что является одним из условий реализации для них децентрализованного управления. Рабочая структура таких систем на системном уровне, задаваемая использованием систем по их целевому назначению, представлена неориентированными графами; ребра графа соответствуют парам дуг взаимно противоположного направления, а диагностические графы реализуются как подграфы рабочих графов. Поэтому анализ свойства изотропности элементарных функций $z$ совместности конкретной модели ненадежного тестирования $M_{01 z z}$ позволяет судить о возможности и целесообразности использования ориентированного или неориентированного диагностических графов.

5.2. Transitivity of elementary functions of zconsistency

Любое бинарное отношение на множестве $V$ является:

- транзитивным $(T)$, когда

$$
\forall v, w, u \in V\{(v T w) \wedge(w T u) \rightarrow(v T u)\},
$$

— интранзитивным $(\operatorname{In} T)$, когда

$$
\forall v, w, u \in V\{(v T w \wedge w T u \rightarrow \overline{(v T u)}\},
$$

— нетранзитивным $(N T)$, когда оно не является транзитивным и не является интранзитивным:

$$
\overline{\forall v, w, u \in V}\{(v T w) \wedge(w T u) \rightarrow(v T u)\} .
$$

Рассмотрим свойство транзитивности применительно к взаимосвязи состояний вершин подграфа тестирования $D_{z}, z \in\{0,1\}$. В табл. 3 приведены результаты анализа отношения транзитивности для элементарных функций zсовместности и дана характеристика задаваемого этими функциями отношения порядка.

\begin{tabular}{|c|c|c|c|}
\hline \multicolumn{2}{|r|}{ ЭФС } & Транзитивность & $\begin{array}{c}\text { Характер отношения } \\
\text { порядка }\end{array}$ \\
\hline 0-I & $f_{0}(v, w)=\bar{v} \bar{w} \vee \bar{v} w \vee v w$ & $\begin{array}{c}T: \\
\left(v^{\prime} \geq w^{\prime}\right) \wedge\left(w^{\prime} \geq u^{\prime}\right) \rightarrow v^{\prime} \geq u^{\prime}\end{array}$ & $\begin{array}{c}\text { Частичный порядок: } \\
v^{\prime} \geq w^{\prime}\end{array}$ \\
\hline 0 -II & $f_{0}(v, w)=\bar{v} w \vee v w$ & $\begin{array}{c}T: \\
\left(v^{\prime} \geq w^{\prime}: w^{\prime}=0\right) \wedge \\
\wedge\left(w^{\prime} \geq u^{\prime}: u^{\prime}=0\right) \rightarrow v^{\prime} \geq u^{\prime}\end{array}$ & $\begin{array}{c}\text { Частичный порядок: } \\
v^{\prime} \geq w^{\prime}: w^{\prime}=0\end{array}$ \\
\hline 0-III & $f_{0}(v, w)=v w \vee \bar{v} w$ & $\begin{array}{c}T: \\
\left(v^{\prime}=w^{\prime}\right) \wedge\left(w^{\prime}=u^{\prime}\right) \rightarrow\left(v^{\prime}=u^{\prime}\right)\end{array}$ & $\begin{array}{c}\text { Эквивалентность: } \\
v^{\prime}=w^{\prime}\end{array}$ \\
\hline 0-IV & $f_{0}(v, w)=v w$ & $\begin{array}{c}T: \\
\left(v^{\prime}=w^{\prime}: w^{\prime}=0\right) \wedge \\
\wedge\left(w^{\prime}=u^{\prime}: u^{\prime}=0\right) \rightarrow v^{\prime}=u^{\prime}=0\end{array}$ & $\begin{array}{l}\text { Эквивалентность: } \\
v^{\prime}=w^{\prime}=0\end{array}$ \\
\hline $1-\mathrm{I}$ & $f_{1}(v, w)=\bar{v} \bar{w} \vee v \bar{w} \vee \bar{v} w$ & $N T: \begin{array}{l}{\left[\left(v^{\prime} \vee w^{\prime}\right)=1\right] \wedge\left[\left(w^{\prime} \vee u^{\prime}\right)=1\right]:} \\
\left(v^{\prime}, w^{\prime}, u^{\prime}\right)=(0,1,0) \rightarrow-\left[\left(v^{\prime} \vee u^{\prime}\right)=1\right]\end{array}$ & $\begin{array}{c}\text { Пересечение: } \\
\forall v, w \in V\{[a(v, w)= \\
=1] \rightarrow\{v, w\} \cap F \neq \varnothing\}\end{array}$ \\
\hline $1-\mathrm{II}$ & $f_{1}(v, w)=v \bar{w} \vee \bar{v} w$ & $\operatorname{InT:}$ & Неравносильность: \\
\hline
\end{tabular}

\section{Отношения транзитивности и порядка для ЭФС.}

Таблица 3 


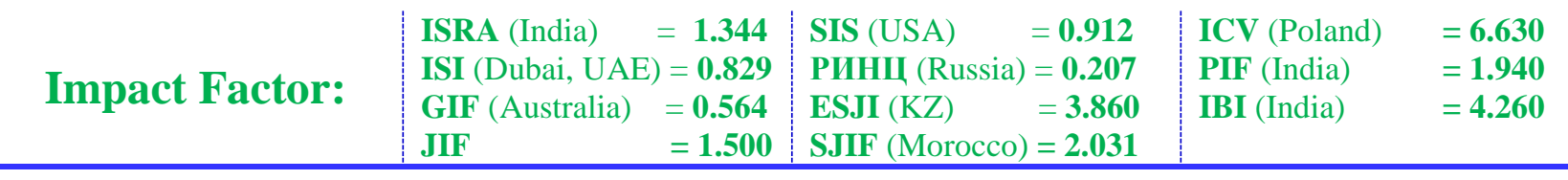

\begin{tabular}{|c|c|c|c|}
\hline & & $\begin{array}{c}{\left[\left(v^{\prime} \neq w^{\prime}\right)\right] \wedge\left[\left(w^{\prime} \neq u^{\prime}\right)\right] \rightarrow} \\
\rightarrow-\left[\left(v^{\prime} \neq u^{\prime}\right)\right]\end{array}$ & $\begin{array}{l}\forall v, w \in V\{[a(v, w)= \\
=1] \rightarrow\left\{v^{\prime} \neq w^{\prime}\right\}\end{array}$ \\
\hline 1-III & $f_{1}(v, w)=\bar{w}=\overline{v w} \vee \bar{w}$ & $\begin{array}{c}T: \\
{\left[\left(v^{\prime} \leq w^{\prime}\right) \wedge\left(w^{\prime} \leq u^{\prime}\right)\right]: w^{\prime}=1 \rightarrow} \\
\rightarrow v^{\prime} \leq u^{\prime}\end{array}$ & $\begin{array}{c}\text { Частичный порядок: } \\
v^{\prime} \leq w^{\prime}: w^{\prime}=1\end{array}$ \\
\hline 1-IV & $f_{1}(v, w)=\bar{w}$ & $\begin{array}{c}N T: \\
(v \bar{w}) \cdot(w \bar{u})=0\end{array}$ & $\begin{array}{c}\text { Любой 1-подгаф-это } \\
\text { тривиальный граф }\end{array}$ \\
\hline
\end{tabular}

Нетранзитивность функции 1-I покажем на примере 1-подграфа, индуцируемого множеством тестов $\left\{\left(v_{1}, v_{2}\right),\left(v_{2}, v_{3}\right)\right\}$. Для него функция 1совместности имеет следующий вид.

$$
\begin{aligned}
& \left(\overline{v_{1}} \vee \overline{v_{2}}\right)\left(\overline{v_{2}} \vee \overline{v_{3}}\right)=\overline{v_{1}} \overline{v_{2}} \vee \overline{v_{1}} \overline{v_{3}} \vee \overline{v_{2}} \vee \overline{v_{2}} \overline{v_{3}}= \\
& =\overline{v_{1}} \overline{v_{2}} \overline{v_{3}} \vee \overline{v_{1}} \overline{v_{2}} v_{3} \vee \overline{v_{1}} v_{2} \overline{v_{3}} \vee \overline{v_{1}} \overline{v_{2}} \overline{v_{3}} \vee v_{1} \overline{v_{2}} v_{3} \Rightarrow \overline{v_{1}} \overline{v_{3}} \vee \overline{v_{1}} v_{3} \vee v_{1} \overline{v_{3}} \vee v_{1} v_{3}
\end{aligned}
$$

Из этого выражения видно, что, хотя состояние $v_{1} \overline{v_{2}} v_{3}$ допустимо для рассматриваемого подграфа 1-совместности, но для пары $\{1,3\}$ состояние вершин 1 и 3 несовместно с исходом $a(1,3)=1$.

Функция 1-II интранзитивна и указывает на несовпадение состояния вершин, участвующих в тестировании. Эта функция является частичной функцией функции 1-I, следовательно, она задает отношение пересечения, но частного вида. Кроме того, исходя из того, что вершины диагностического графа имеют два финальных состояния - исправная и неисправная функция 1-II задает отношение эквивалентности особого вида:

$$
\forall v, w, u \in V\left\{(v \operatorname{InTw}) \wedge(w \operatorname{InTu}) \rightarrow v^{\prime}=u^{\prime}\right\}
$$

- отношение неравносильности для смежных вершин, или деление вершин 1-компонент связности на классы неравносильности.

Проиллюстрируем примерами полезность использования теоретико-множественного подхода при анализе значимости элементарных функций $z$-совместности.

П р и м е $\mathrm{p}$ 2. Функции 0-I, 0-II и 1-III задают отношение частичного порядка. Для модели $M_{01 \times x}$ известно свойство предшествования неисправных вершин исправным в простом 0 -пути. В ряде работ это свойство доказывается с помощью цепочек суждений, касающихся анализа совместности синдрома с порождающими его состояниями вершин. Однако это свойство можно вывести как прямое следствие отношения частичного порядка, установленного для функции 0-I. Для функции 0-II, являющейся частью функции 0-I, свойство предшествования принимает следующую форму: в простом 0 -пути имеется не более чем одна неисправная вершина начальная вершина пути. Функция 1-III является двойственной к функции 0-II; следовательно, для нее имеет место свойство предшествования исправной вершины неисправным: в простом 1пути имеется не более чем одна исправная вершина - начальная вершина пути.

П р и м е p 3. Функции 0-III и 0-IV задают отношение эквивалентности, при котором в одном классе эквивалентности оказываются элементы, неразличимые с точки зрения некоторого отношения $R$. Следовательно, класс эквивалентности определяется любым своим представителем, т.е. произвольным элементом этого класса. Применительно к самодиагностированию сказанное означает, что 1) отношение $R$, выполняемое для некоторого $D_{0}^{(i)}=\left(V_{0}^{(i)}, E_{0}^{(i)}\right)$, имеет место для всяких $v \notin V_{0}^{(i)}$ и $w \in V_{0}^{(i)}$, таких, что $(v, w) \in E$ или $(w, v) \in E$, если на очередном $((i+1)$-м) шаге самодиагностирования будет получено $a(v, w)=0$ или $a(w, v)=0$ соответственно; 2) вместо изучения отношений между классами эквивалентности достаточно изучить отношения между их представителями.

\section{Conclusion}

Решается задача самодиагностирования живучих многопроцессорных вычислительных систем в присутствии кратных устойчивых неисправностей, рассматриваемых на уровне (процессорных) модулей, и при использовании полных ненадежных тестов. Самодиагностирование основано на сопоставительном анализе исходов тестирования одних модулей системы другими. Решение задачи самодиагностирования рассматривается в рамках развиваемого в работах автора подхода, когда состояние модулей определяется по результатам сопоставительного анализа исходов тестирования, относящихся к их ограниченной окрестности и осуществляется в процессе 
диагностирования с помощью децентрализованных адаптивных алгоритмов.

Для решения задачи самодиагностирования использована введенная в работах автора обобщенная структурированная теоретикографовая модель самодиагностики, вычленяющая из классической модели [1] компоненту, связанную с описанием свойств используемых полных ненадежных тестов. Применение обобщенной модели позволило с единых позиций анализировать и сравнивать диагностические свойства разных моделей ненадежных тестов из исчерпывающей группы известных моделей [4].

В качестве единого формального аппарата для описания и анализа свойств ненадежных тестов использованы булевы функции (функции совместности), представление которых адаптировано к решению задачи самодиагностирования. Выделено базовое множество функций совместности (элементарные функции совместности), сочетания которых описывают модели ненадежных тестов из группы [4]. Введены характеристики значимости функций совместности относительно возможности 1) идентифицировать с их помощью фактическое состояние модулей вычислительной системы в ходе выполнения локального самодиагностирования и 2) выбирать последовательность тестирования соответственно результатам выполненного тестирования.
Применив теоретико-множественный подход, изучена значимость элементарных функций совместности, характеризуемая соответствием между отношением порядка модулей по их роли в выполнении теста (тестирующий-тестируемый) и отношением порядка модулей по их техническому состоянию (исправен-неисправен). При этом, в частности, описан вид симметрии булевых функций, по аналогии с физикой названный "изотропностью." Для изотропных элементарных функций совместности исход теста зависит только от состояния участвующих в нем модулей и инвариантен к их роли (тестируемыйтестирующий) в тесте. Показана связь изотропности со свойством ориентированности используемых рабочего и диагностического графов многопроцессорной вычислительной системы.

Полученные результаты дают основание для постановки новой задачи, являющейся частью задачи локального самодиагностирования: анализу свойств значимости функций совместности, описывающих подграфы тестирования, которые могут образовываться в заданном диагностическом графе в процессе самодиагностирования. Результаты такого анализа могут быть полезны не только для разработки эффективных алгоритмов локального самодиагностирования, но и при решении задач анализа и синтеза диагностического графа.

\section{ПРИЛОЖЕНИЕ}

\section{Список используемых обозначений}

$|X|$ - мощность множества $X$

$\lfloor x\rfloor$ - наибольшее целое такое, что $\lfloor x\rfloor \leq x$

$$
D=(V, E) \text { - ориентированный граф с }
$$
взвешенными дугами, представляющий диагностическую структуру системы; диагностический граф.

$$
V=\{v: v \in V\}-\text { множество вершин, }
$$

представляющих модули системы; $|V|=N$ число модулей системы.

$$
E=\{(v, w): \quad v, w \in V\}-\text { множество дуг, }
$$
представляющих тесты - связи от тестирующей вершины $(v)$ к тестируемой вершине $(w)$.

$$
a(v, w) \in\{\varnothing, 0,1\} \quad \text { - вес дуги }(v, w) \text {; }
$$
$a(v, w)=\varnothing$, если тест $(v, w)$ не выполнен, $a(v, w)=0$, если тест $(v, w)$ выполнен и вершина $v$ считает вершину $w$ исправной и $a(v, w)=1$, если тест $(v, w)$ выполнен и вершина $v$ считает вершину $w$ неисправной.

$t$ - кратность неисправностей; $t \leq N$.

$F \subset V$ - образ неисправностей: множество одновременно неисправных вершин графа.

$$
F(t)=\left\{F_{k}: \quad\left|F_{k}\right| \leq t ; \quad k=\overline{1,|F(t)|}\right\}
$$

множество допустимых образов неисправностей.

$$
\sigma(F)=\{a(v, w) \mid(v, w) \in E\}
$$

упорядоченное множество весов дуг диагностического графа - синдром состояния графа, совместный с заданным образом неисправностей $F$ (порождаемый $F$ ).

$$
\Gamma^{-1}(v)=\{w: \quad(w, v) \in E\} \quad-\quad \text { множество }
$$
вершин, тестирующих вершину $v$.

$$
\Gamma(v)=\{w: \quad(v, w) \in E\} \quad-\quad \text { множество }
$$

вершин, тестируемых из вершины $v$. 


\begin{tabular}{l|lrl|l|ll} 
& ISRA (India) & $=\mathbf{1 . 3 4 4}$ & SIS (USA) & $=\mathbf{0 . 9 1 2}$ & ICV (Poland) & $=\mathbf{6 . 6 3 0}$ \\
Impact Factor: & ISI (Dubai, UAE) $=\mathbf{0 . 8 2 9}$ & PVHU (Russia) $=\mathbf{0 . 2 0 7}$ & PIF (India) & $=\mathbf{1 . 9 4 0}$ \\
& GIF (Australia) & $\mathbf{0 . 5 6 4}$ & ESJI (KZ) & $=\mathbf{3 . 8 6 0}$ & IBI (India) & $\mathbf{4 . 2 6 0}$
\end{tabular}

$$
\begin{gathered}
\text { Для } \quad X \subset V: \quad \Gamma^{-1}(X)=\left\{\bigcup_{v \in X} \Gamma^{-1}(v)-X\right\} ; \\
\Gamma(X)=\left\{\bigcup_{v \in X} \Gamma(v)-X\right\} .
\end{gathered}
$$

\section{References:}

1. Preparata F.P., Metze G., Chien R.J. (1967) On Connection Assignement Problem of Diagnosable Systems // IEEE Trans. El. Comput. 1967. V. EC-16. No. 12. P. 848-854.

2. Dimitriev Yu.K. (2007) Lokal'noe samodiagnostirovanie v vychislitel'nyh sistemah s cirkulyantnoj strukturoj // Avtomatika i telemekhanika. 2007. № 3. P. 187-198.

3. Dimitriev Yu.K. (2007) Local Diagnostication in Computer Systems with the Circulant Structure // Autom. Remote Control. 2007. V. 68. No. 3. P. 545-556.

4. Dimitriev Yu.K. (2012) Pravila uslovnogo lokal'nogo samoopredeleniya i algoritm diagnostirovaniya mul'tiprocessornoj sistemy $\mathrm{s}$ cirkulyantnoj diagnosticheskoj strukturoj na ih osnove // Avtomatika i telemekhanika. 2012. № 5. P. $125-140$.

5. Dimitriev Yu.K. (2012) Conditional Local Selfregulation Rules and a Diagnostic Algorithm for a Multiprocessor System with Circulant Diagnostic Structure Based on These Rules // Autom. Remote Control. 2012. V. 73. No. 5. P. 862-872.

6. Kavianpour A., Friedman A. (1980) A Different Diagnostic Models for Multiprocessor Systems // Proc. Inf. Processing-80 (IFIP Congress 80). Tokyo-Melburn. 1980. P. 157-162.

7. Dimitriev Yu.K. (2015) O konceptual'noj osnove sravnitel'nogo analiza i resheniya zadach samodiagnostiki mnogoprocessornyh sistem dlya raznyh modelej nenadezhnogo testirovaniya // Avtomatika i telemekhanika. 2015. № 7. P. 150-164.
8. Dimitriev Yu.K. (2015) On the Conceptual Foundations of Comparative Analysis and Solution of Self-Diagnostic Problems in Multiprocessor Systems under Different Unreliable Testing Models // Autom. Remote Control. 2015. V. 76. No. 7. P. 1260-1270.

9. Dimitriev Yu.K. (2008) Chastichnoe i polnoe lokal'noe diagnostirovanie $\mathrm{v}$ vychislitel'nyh sistemah s cirkulyantnoj strukturoj. // Avtomatika i telemekhanika. 2008. №7. P. 136-145.

10. Dimitriev Yu.K. (2008) Partial and Complete Local Diagnosis in Computer Systems with Circulant Structure // Autom. Remote Control. 2008. V. 69, No. 7. P. 1223-1230.

11. Dimitriev Yu.K. (2010) Analiz vozmozhnosti lokal'nogo diagnostirovaniya $\mathrm{v}$ vychislitel'nyh sistemah s cirkulyantnoj strukturoj na osnove ispol'zovaniya izbytochnosti $\mathrm{v}$ chisle analiziruemyh iskhodov testirovaniya // Avtomatika i telemekhanika. 2010. № 4. P. 169-180.

12. Toffoli T., Margolus N. (1991) Mashiny kletochnyh avtomatov / Per. s angl. M.: "Mir".1991. 280 p. ISBN 5-03-001619-8

13. Dimitriyev Yu.K. (1993) Samodiagnostika modulnykh vychislitelnykh sistem // VO "Nauka". Novosibirsk. $1993-293$ p. ISBN 5-02-030326-7

14. Paulin O.N. (2005) K postroeniyu prikladnoj teorii simmetricheskih bulevyh funkcij // Nauchno-tekhnicheskij zhurnal «Iskusstvennyj intellekt» (Ukraina). 2005. №4. P. 245-255. ISSN 1561-5367. 